\title{
Application of In-situ TEM Nanoscale Quantitative Mechanical Testing to Elastomers
}

Christopher M. Barr ${ }^{*}$, Dan Qu², William M. Mook ${ }^{1}$, Bryan Korth ${ }^{2}$, Jamie M. Kropka ${ }^{1}$, Craig Burkhart ${ }^{2}$, Khalid Hattar ${ }^{1}$

1. Sandia National Laboratories, Albuquerque, NM USA

2. The Goodyear Tire \& Rubber Company, Akron, OH USA

* Corresponding author: cbarr@sandia.gov

The utilization of in-situ TEM quantitative nanomechanical testing for metallic and ceramic materials has been widely successful in elucidating active deformation mechanisms under a range of loading and sample geometries [1]. Advances more recently in mechanical testing capabilities in the TEM include adding concurrent or sequential additional external stimuli including heating, laser and ion irradiation [2, 3], local strain analysis [4], and dynamic mechanical testing modes [5]. The success of these small scale in-situ TEM testing capabilities, however, has not been as widely adopted for polymer and soft materials. The notable challenges for in-situ TEM mechanical testing of polymers in the TEM include highly specialized sample preparation, electron beam irradiation, oxidation, and challenging specimen fabrication. In this study, we attempt to overcome these barriers by developing an adaptable sample preparation and testing protocol for small scale mechanical testing of elastomers in the TEM.

To develop a method for evaluating the nanoscale mechanical response of elastomers in the TEM, bulk elastomers and polymer matrix composites were examined from The Goodyear Tire \& Rubber Company. Cryo-ultramicrotome was utilized to create thin films of the elastomer material with varying thickness between 200 and $600 \mathrm{~nm}$. The elastomer thin films were then transferred and aligned to Bruker Hysitron push-to-pull devices, as shown in Figure 1a. Following this manual alignment of the elastomer thin film onto the PTP devices, a $30 \mathrm{kV}$ Ga FIB was used to fabricate a small tensile specimen, shown in Figure $1 \mathrm{~b}$, for evaluation in the TEM. Monotonic tensile tests were performed in a JEOL $2100 \mathrm{LaB}_{6}$ microscope at $120 \mathrm{kV}$ using a Bruker Hysitron PI-95 stage [3]. Between all preparation steps, samples were stored in a dry $\mathrm{N}_{2}$ backfilled environment to minimize any deleterious oxidation. To evaluate the effect of electron beam irradiation, thin samples of the elastomers were also prepared on standard TEM mesh grids and irradiated at various electron energies, dose, and dose rates.

The in-situ TEM mechanical thin film fracture morphology is shown in Figure 2 for a typical elastomer sample. In-situ TEM quantitative stress-strain behavior show that the deformation behavior is significantly different than bulk scale elastomer performance. The presentation will discuss proposed causes for the apparent lack of traditional elastomeric deformation behavior in these small-scale tests. Finally, a perspective is given on the adaption of these in-situ mechanical test for polymer, polymer matrix composites, and other soft or biological materials with emphasis on electron dose effects and role of possible chemical and structural sample preparation modifications [6]. 


\section{References:}

[1] A.M. Minor, S.A. Asif, Z. Shan, E.A. Stach, E. Cyrankowski, T.J. Wyrobek, O.L. Warren, Nature Mateirals 5 (2006) 697-702.

[2] G.S. Jawaharram, P.M. Price, C.M. Barr, K. Hattar, R.S. Averback, S.J. Dillon, Scripta Materialia 148 (2018) 1-4.

[3] K. Hattar, D.C. Bufford, D.L. Buller, Nuclear Instruments and Methods in Physics Research Section

B: Beam Interactions with Materials and Atoms 338 (2014) 56-65.

[4] C. Gammer, C. Ophus, T.C. Pekin, J. Eckert, A.M. Minor, Applied Physics Letters 338 (2018) 112.

[5] D.C. Bufford, D. Stauffer, W.M. Mook, S.A. Syed Asif, B.L. Boyce, K. Hattar, Nano Letters 16 (2016) 4946-4953.

[6] Sandia National Laboratories is a multi-mission laboratory managed and operated by National Technology \& Engineering Solutions of Sandia, LLC., a wholly owned subsidiary of Honeywell International, Inc., for the U.S. Department of Energy's National Nuclear Security Administration under contract DE-NA0003525.
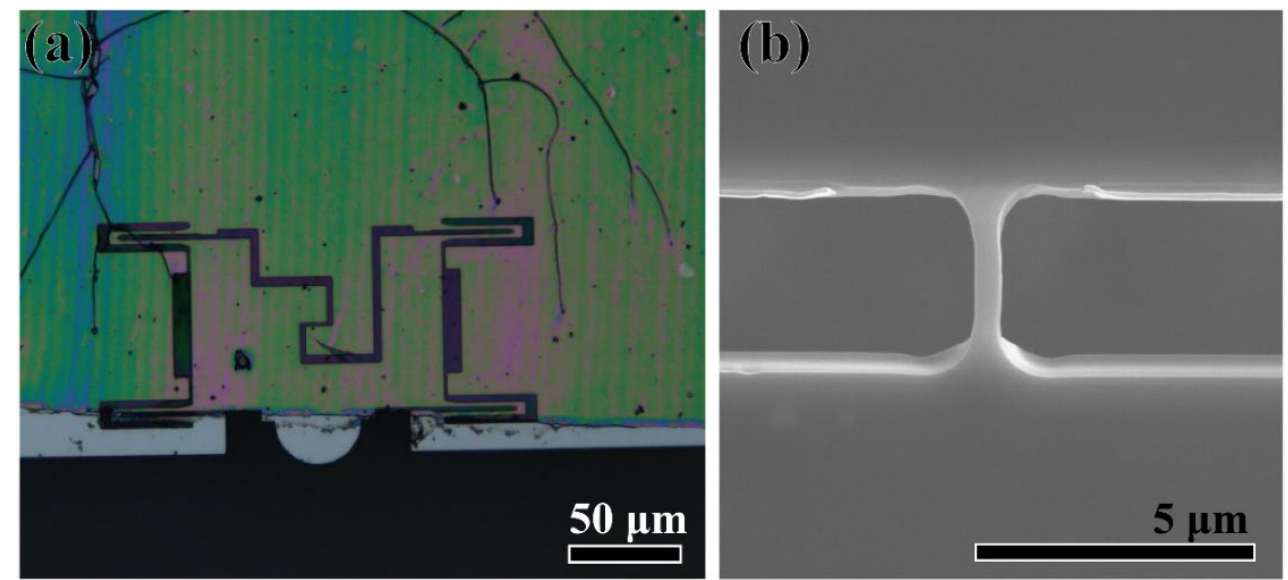

Figure 1. (a) optical microscope image of elastomer thin sheet on push-to-pull device; (b) FIB prepared elastomer tension specimen in the center of the push-to-pull device

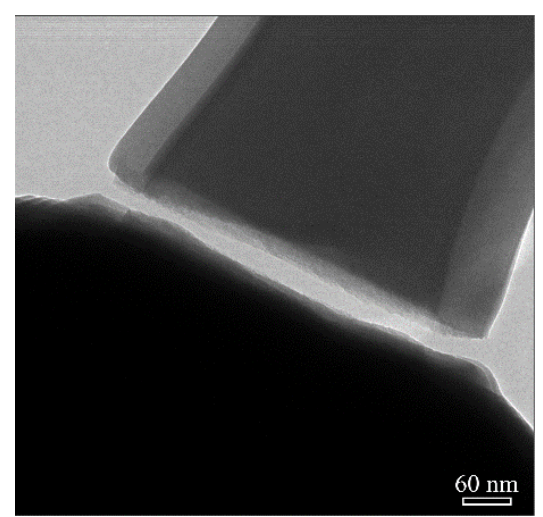

Figure 2. Typical fracture morphology of the elastomer thin films after an in-situ TEM monotonic loadcontrolled test 